\title{
Frontières
}

\section{Suicide, hommes et socialisation}

\section{Lucie Charbonneau et Janie Houle}

Volume 12, numéro 1, automne 1999

Suicides, générations et culture

URI : https://id.erudit.org/iderudit/1074510ar

DOI : https://doi.org/10.7202/1074510ar

Aller au sommaire du numéro

Éditeur(s)

Université du Québec à Montréal

ISSN

1180-3479 (imprimé)

1916-0976 (numérique)

Découvrir la revue

\section{Citer cet article}

Charbonneau, L. \& Houle, J. (1999). Suicide, hommes et socialisation. Frontières, 12(1), 62-68. https://doi.org/10.7202/1074510ar

\section{Résumé de l'article}

Depuis les années 70, au Québec, le taux de suicide des hommes a grimpé de $78 \%$ et aujourd'hui, $80 \%$ des suicides sont commis par des hommes. Pourquoi les hommes se suicident-ils plus que les femmes ? Plusieurs éléments contribuent à cette surmortalité, mais la socialisation masculine apparaît comme leur dénominateur commun. En effet, la socialisation masculine facilite l'apparition de facteurs de risque liés au suicide et empêche l'émergence de facteurs de protection. Comme société et comme individus, il nous faut réfléchir à nos pratiques et à nos attitudes face aux hommes en difficulté afin de créer un espace où leur souffrance pourra s'exprimer. 


\section{Résumé}

Depuis les années 70, au Québec, le taux de suicide des hommes a grimpé de $78 \%$ et aujourd'hui, $80 \%$ des suicides sont commis par des hommes. Pourquoi les hommes se suicident-ils plus que les femmes? Plusieurs éléments contribuent à cette surmortalité, mais la socialisation masculine apparaît comme leur dénominateur commun. En effet, la socialisation masculine facilite l'apparition de facteurs de risque liés au suicide et empêche l'émergence de facteurs de protection. Comme société et comme individus, il nous faut réfléchir à nos pratiques et à nos attitudes face aux hommes en difficulté afin de créer un espace où leur souffrance pourra s'exprimer.

Mots clés: suicide - hommes - Québec facteurs de risque - socialisation

\begin{abstract}
In Québec the male suicide rate has increased by $78 \%$ since the 1970's and men today commit $80 \%$ of suicides. Why do men commit suicide more than women? Many variables contribute to this comparatively higher death rate, yet male socialization seems to be a common denominator. Male socialization encourages certain risk factors linked to suicide to appear and inhibits certain protection factors from emerging. As a society and as individuals, we need to consider our practices and our attitudes towards troubled men in order to create a space in which their suffering can be expressed.
\end{abstract}

Key words: suicide - men - Québec - risk factors - socialization

\section{SUICIDE, HOMMES ET SOCIALISATION}

\author{
Lucie Charbonneau, \\ responsable de projets \\ à I'Association québécoise de suicidologie, \\ membre du CRISE. \\ Janie Houle, \\ doctorante en psychologie à I'UQAM, \\ membre du CRISE.
}

Pourquoi les hommes se suicident-ils quatre fois plus que les femmes? Que pouvons-nous faire pour mieux intervenir auprès d'eux et contribuer ainsi à atténuer la souffrance et le désespoir qui les amènent à s'enlever la vie? Cet article tente de mieux comprendre ce phénomène qui affecte toute la population québécoise et vise à amorcer une réflexion sur nos pratiques et nos attitudes face aux hommes en difficulté. D'abord, il présente un portrait statistique du suicide au Québec, pour ensuite proposer une synthèse des principaux facteurs pouvant expliquer cette plus grande incidence de suicide chez les hommes. Tout en étant conscientes du fait que le suicide est multidéterminé, les auteurs accordent une place privilégiée à la socialisation masculine dans l'explication de la différence importante dans les taux de suicide des hommes et des femmes. La troisième partie de l'article se penche sur la question de l'intervention auprès des clientèles masculines.

\section{QUELQUES FAITS}

Depuis les deux dernières décennies, le taux de suicide a connu une progression constante. Entre 1976 et 1996, il a augmenté de $62 \%$ dans la population totale. Chez les femmes, il est passé de 6,8 à 8,5 pour 100000 personnes, tandis que chez les hommes, il est passé de 17,4 à 31,0 pour 100000 personnes, soit des augmentations respectives de $25 \%$ et de $78 \%$ (voir Tableau 1). Cette augmentation majeure a fait de cette cause de mortalité un problème préoccupant de santé publique, pour ne rien dire du problème social que cela soulève ${ }^{1}$.

En 1997, 1003 hommes sont décédés par suicide au Québec, soit près de $80 \%$ de tous les décès par suicide (1 263). Sur l'ensemble des décès par suicide enregistrés chez les hommes, la majorité survient chez ceux âgés de moins de 55 ans, soit près de 8 décès sur 10. En 1997, sur les 1003 décès par suicide chez les hommes, $16 \%$ (161) étaient le fait de jeunes de moins de 24 ans, $64 \%$ (636) le fait d'hommes adultes de 25 à 54 ans et $20 \%$ (206) d'hommes de plus de 55 ans. Ainsi, contrairement à ce qui est souvent véhiculé dans les médias, le suicide chez les hommes adultes n'existe pas uniquement chez les jeunes ou chez les hommes âgés. Cependant, si davantage de décès sont 
enregistrés en nombre absolu chez les hommes adultes, les jeunes présentent aussi des taux de mortalité très élevés. D'ailleurs, les taux de mortalité les plus élevés sont observés chez les hommes de 20 à 44 ans $^{2}$.

Pour ce qui est des idéations suicidaires et des tentatives de suicide, les données rapportent plus fréquemment ces comportements chez les femmes que chez les hommes. Canetto et Sakinosfski s'interrogent sur l'exactitude de ces données ${ }^{3}$. Suite à une revue des taux provenant de différents pays, ils notent que la surreprésentation des femmes dans les conduites suicidaires non fatales n'est pas un phénomène aussi uniforme que prévu. Ils concluent que le rapport homme-femme est tributaire du milieu culturel dans lequel il prend place.

Au Québec, les données de l'Enquête Santé Québec indiquent peu de différences entre les hommes et les femmes relativement aux comportements suicidaires non fatals 4 . Au cours de leur vie, $7,4 \%$ des hommes et $8,5 \%$ des femmes indiquent avoir pensé sérieusement à se suicider. L'écart entre hommes et femmes se creuse un peu plus au niveau des tentatives de suicide au cours de la vie. Les données de l'Enquête Santé Québec démontrent que 2,9\% des hommes et $4,2 \%$ des femmes rapportent avoir fait une tentative de suicide au cours de leur vie.

\section{UNE SOCIALISATION PROPICE AU SUICIDE}

La socialisation masculine joue un rôle déterminant dans le suicide des hommes. Comme nous le verrons dans cette deuxième partie, elle exerce son influence, d'une part, en facilitant l'émergence de certains facteurs de risque et, d'autre part, en inhibant cer- tains facteurs de protection. En effet, elle véhicule l'idée que le suicide est plus acceptable chez les hommes que chez les femmes et facilite, par le fait même, l'adoption de ce comportement par les hommes. De plus, la socialisation masculine tolère et encourage l'agressivité et l'impulsivité de même que la consommation d'alcool et de drogue chez les hommes. Or, ces éléments sont tous considérés comme des facteurs de risque importants du suicide. Elle favorise également une plus grande accessibilité aux armes à feu, diminuant ainsi les chances de pouvoir secourir la personne à temps. Par ailleurs, la rigidité du rôle masculin entraîne une stigmatisation sociale qui place l'homosexualité au rang des facteurs de risque de certains comportements suicidaires. Finalement, la socialisation masculine augmente la vulnérabilité des hommes face à la perte d'emploi et à la rupture amoureuse.

En plus d'exercer son influence facilitatrice, la socialisation masculine inhibe l'émergence de facteurs de protection contre le suicide. Elle contribue en effet à réduire l'éventail de mécanismes d'adaptation dont les hommes disposent puisqu'elle valorise l'autonomie et l'indépendance, tout en stigmatisant l'expression de la souffrance et la demande d'aide. Ainsi, en situation de crise, les hommes qui sont coupés des sources potentielles d'aide se privent d'un soutien qui pourrait s'avérer essentiel. De plus, les années postrévolution tranquille semblent offrir de meilleures possibilités d'avenir aux femmes alors qu'elles laissent les hommes dans un flou identitaire.

La socialisation nous apparaît donc comme un dénominateur commun dans la problématique du suicide chez les

Tableau 1

Taux de mortalité par suicide selon le genre

Québec, 1976-1996
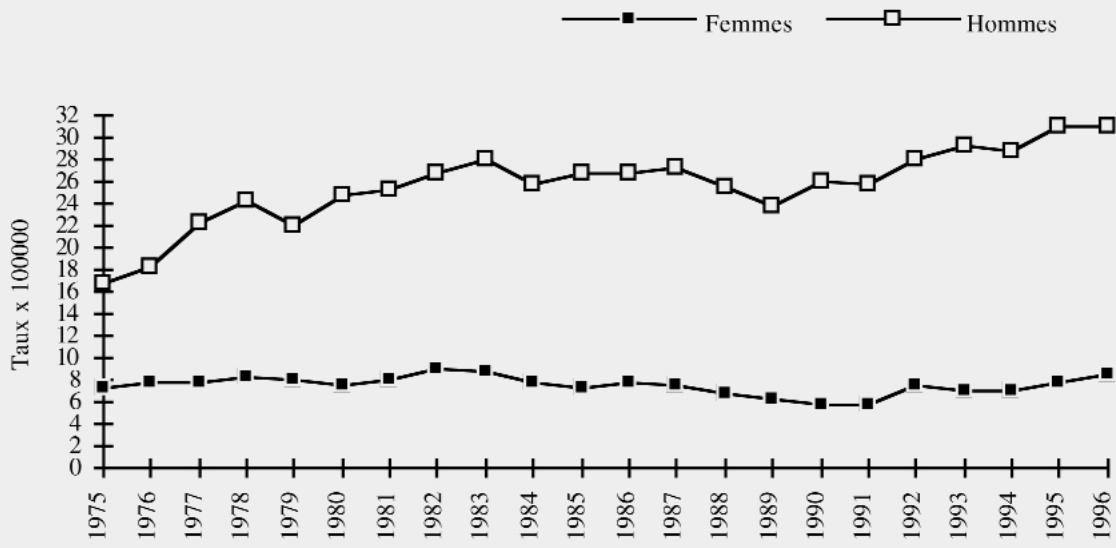

hommes, de par son influence facilitatrice ou inhibitrice auprès des facteurs clés $\mathrm{du}$ processus suicidaire. Examinons maintenant certains des facteurs de risque pouvant permettre d'expliquer cette plus grande incidence du suicide chez la gent masculine.

\section{FACTEURS DE RISQUE DU SUICIDE CHEZ LES HOMMES}

\section{Acceptabilité du suicide}

Le suicide est un comportement généralement désapprouvé socialement mais, néanmoins, dans certaines conditions et pour certaines personnes, considéré comme plus acceptable. Il en est ainsi pour les personnes atteintes de maladie terminale et... pour les hommes. En effet, les tentatives de suicide sont considérées comme plus féminines que le suicide complété ${ }^{5}$, plutôt considéré lui comme un comportement masculin, au même titre que l'abus d'alcool et de drogue ${ }^{6}$. Les gestes suicidaires fatals sont donc tolérés, voire encouragés chez les hommes dans certaines circonstances. On remarque d'ailleurs que les hommes reçoivent moins de sympathie que les femmes à l'égard de leur tentative de suicide ${ }^{7}$ et qu'ils sont plus critiques et inconfortables envers le comportement suicidaire ${ }^{8}$, particulièrement s'il est le fait d'un autre homme ${ }^{9}$.

Ainsi, en raison de l'association entre les tentatives de suicide et la féminité, les hommes craignent davantage la désapprobation sociale associée aux actes suicidaires non fatals que les femmes ${ }^{10}$ et ils seraient plus enclins à compléter leur suicide pour s'y soustraire. Les personnes qui pensent au suicide prennent donc en considération ce que les gens vont penser d'eux, suite à leur passage à l'acte, et ces inférences jouent un rôle déterminant dans le processus de prise de décision ${ }^{11}$. Les hommes et les femmes vont tendre à adopter les comportements suicidaires qui sont cohérents avec les scénarios culturels véhiculés par l'idéologie dominante et qui correspondent à leur genre ${ }^{12}$. D'ailleurs, les anthropologues ont depuis longtemps reconnu que le comportement suicidaire était modelé culturellement ${ }^{13}$, d'autant plus que la corrélation entre les scénarios culturels et l'épidémiologie est une constante entre les différentes cultures et les différentes époques ${ }^{14}$.

Les hommes québécois se conforment aux scénarios qui prévalent actuellement dans la société nord-américaine, à savoir que le suicide est un comportement plus acceptable pour eux et qu'y survivre se paie chèrement d'un stigma social 


\section{L'agressivité et l'impulsivité}

Dans plusieurs cultures, dont la nôtre, l'agressivité masculine fait l'objet d'une grande tolérance qui se reflète dans la prévalence des comportements agressifs plus fréquents chez les hommes. À cet égard, certaines études transculturelles montrent clairement que les normes sociales en matière d'agressivité, qui diffèrent d'une société à l'autre, sont fidèlement reproduites dans l'épidémiologie des comportements agressifs de cette société15.

L'agressivité excessive et non contrôlée est fréquemment observée chez les personnes suicidaires ${ }^{16}$. Il en est de même pour l'impulsivité dans les comportements violents qui est associée au comportement suicidaire chez les adolescents $^{17}$, les alcooliques ${ }^{18}$, les personnes dépressives ${ }^{19}$ et les personnes hospitalisées en psychiatrie ${ }^{20}$.

Bien que plusieurs études s'entendent pour reconnaître l'importance déterminante des facteurs biologiques dans l'agressivité et l'impulsivité, il n'en demeure pas moins que la socialisation joue un rôle de facilitateur ou d'inhibiteur et qu'il doit être considéré dans l'équation. Considérant l'ensemble de ces évidences, il semble raisonnable de prétendre que l'agressivité et l'impulsivité dont font preuve les hommes, notamment en raison de la façon dont ils sont socialisés, contribuent certainement à favoriser chez eux une plus grande vulnérabilité au suicide.

\section{Utilisation des moyens létaux}

$\mathrm{Au}$ Québec, les hommes privilégient des moyens différents des femmes pour se suicider. Comme on le remarque au Tableau 2, bien que l'utilisation de l'arme à feu ait diminué autant chez les hommes que chez les femmes, il n'en demeure pas moins que les hommes utilisent des méthodes de suicide plus létales que les femmes (pendaison et arme à feu vs intoxication).

Cette prédominance de l'utilisation des armes à feu et de la pendaison par les hommes ne serait pourtant pas exclusivement le fruit des différences sexuelles, mais également le résultat d'influences culturelles plus larges. En effet, il semble que l'acte de s'enlever la vie soit régi par un scénario requérant l'utilisation d'une méthode particulière, déterminée par le système de valeurs culturelles, le sexe et l'âge de la personne ${ }^{21}$.

Lorsqu'on compare deux pays très rapprochés comme les États-Unis et le Canada, on observe clairement l'influence de la culture dans le choix de la méthode de suicide. Aux États-Unis, où

Tableau 2

Répartition des méthodes de suicide Québec, 1989-1991 et 1992-1994

\begin{tabular}{|c|c|c|c|c|}
\hline & $\begin{array}{r}\text { Hommes } \\
1989-1991\end{array}$ & 1992-1994 & $\begin{array}{r}\text { Femmes } \\
1989-1991\end{array}$ & 1992-1994 \\
\hline Pendaison & $37 \%$ & $40 \%$ & $26 \%$ & $31 \%$ \\
\hline Arme à feu & $32 \%$ & $27 \%$ & $12 \%$ & $7 \%$ \\
\hline Intoxication CO & $11 \%$ & $12 \%$ & $8 \%$ & $11 \%$ \\
\hline Autres intoxications & $7 \%$ & $7 \%$ & $28 \%$ & $24 \%$ \\
\hline Chute & $5 \%$ & $5 \%$ & $9 \%$ & $7 \%$ \\
\hline Noyade & $3 \%$ & $3 \%$ & $8 \%$ & $8 \%$ \\
\hline Autres & $5 \%$ & $6 \%$ & $9 \%$ & $12 \%$ \\
\hline lotal & $100 \%$ & $100 \%$ & $100 \%$ & $100 \%$ \\
\hline
\end{tabular}

les armes à feu sont disponibles et socialement acceptées, elles représentent la méthode la plus utilisée par les deux sexes, particulièrement par les hommes $^{22}$. Au Canada, où il n'y a pas la même acceptabilité à l'égard de la possession d'arme, ce moyen est beaucoup moins utilisé pour se suicider.

En somme, les hommes et les femmes utilisent les méthodes qui leur sont accessibles, familières et acceptables en tant qu'homme et femme dans leur communauté23. Au Québec, les moyens les plus létaux sont l'apanage de la gent masculine. Cette plus grande létalité des moyens contribue à l'incidence beaucoup plus élevée du suicide chez les hommes.

\section{La consommation d'alcool et de drogue}

Les hommes et les femmes ne présentent pas les mêmes configurations de troubles psychologiques. Les femmes sont plus susceptibles de souffrir de dépression et de troubles anxieux, alors que les hommes sont davantage affectés par les troubles d'abus de substances (alcool et drogue) et les troubles de personnalité ${ }^{24}$. Or, il est fort probable que la socialisation conduise les hommes et les femmes à exprimer leur détresse de façon différente et qu'elle soit responsable de cette spécificité sexuelle des problèmes de santé mentale ${ }^{25}$. Sylvia Canetto avance même l'idée que l'abus de substances pourrait être l'alternative masculine à la tentative de suicide, puisque cette dernière est trop stigmatisante pour les hommes ${ }^{26}$.
$\mathrm{Au}$ Québec, les données sont unanimes: les hommes consomment beaucoup plus d'alcool que les femmes. L'Enquête sociale et de santé 1992-1993 indique que les hommes sont beaucoup plus nombreux que les femmes à faire partie des deux catégories de buveurs les plus à risque de développer des problèmes sociaux liés à l'alcool, c'est-àdire celle des buveurs occasionnellement excessifs (15\% vs. $5 \%)$ et celle des grands buveurs $(2.5 \% \text { vs. } 0.2 \%)^{27}$. Le calcul d'un indice de risque des problèmes liés à l'alcool a également permis de constater que les hommes sont près de trois fois plus nombreux que les femmes à présenter un risque élevé (19\% vs 7\%). Cet indice est particulièrement évocateur de l'importance de la consommation d'alcool dans la problématique du suicide puisqu'il est fortement associé à la détresse psychologique, aux idéations suicidaires et aux tentatives de suicide. En outre, les troubles d'abus et de dépendance à l'alcool et aux drogues sont présents dans 30 à $50 \%$ des cas de suicide. On a noté que plus du tiers des personnes qui se suicident avaient de l'alcool dans le sang au moment du décès ${ }^{28}$. Les hommes de 45 à 50 ans, qui consomment en moyenne depuis 19 ans, représentent le groupe le plus à risque de se suicider.

La consommation d'alcool est souvent utilisée par les hommes comme une forme d'automédication, une façon de gérer leur souffrance. Bien que cette stratégie procure un soulagement ponctuel de leur détresse, elle entraîne néanmoins des effets négatifs, d'une part, en 
induisant un état dépressif après une période de consommation chronique et, d'autre part, en contribuant à faciliter le passage à l'acte suicidaire dans les moments de découragement.

\section{Le chômage et le surinvestissement des hommes dans les sphères du travail}

L'association entre le taux de chômage et les taux de suicide et de tentatives de suicide est depuis longtemps reconnue ${ }^{29}$. Il semble cependant que les hommes soient plus vulnérables à ce facteur de risque que ne le sont les femmes. Traditionnellement, l'homme occidental se valorise par son travail ${ }^{30}$. Encore aujourd'hui, ses activités professionnelles tiennent une place importante dans sa vie ${ }^{31}$. Son identité et son estime de soi sont fortement influencées par ses performances dans la sphère du travail parce que le métier qu'il exerce est central dans sa perception de lui-même ${ }^{32}$. L'échec dans le domaine du travail et les impacts du chômage seront plus dévastateurs pour les hommes que pour les femmes puisque celles-ci dépendent moins de leur statut d'emploi pour se valoriser et s'accomplir ${ }^{33}$. Il semble donc que, dans le contexte économique et social actuel, le surinvestissement des hommes dans la sphère $d u$ travail contribue à leur plus grande vulnérabilité au suicide.

\section{Le statut matrimonial}

\section{et la rupture amoureuse}

De 1951 à 1986, au Québec et dans les autres provinces canadiennes, le taux de suicide a augmenté parallèlement à la hausse du taux du divorce. Au niveau épidémiologique, les hommes divorcés et les veufs se suicident plus souvent que les hommes mariés, parfois même jusqu'à six fois plus $^{34}$. La différence est beaucoup moins grande chez les femmes parce que, dans la majorité des cas, ce sont les femmes qui ont la garde des enfants et qu'elles ont des liens plus forts avec leur famille et leurs amies, pouvant ainsi plus facilement satisfaire leurs besoins émotifs en dehors d'une relation conjugale ${ }^{35}$. D'autre part, la conjointe se révèle souvent être la seule confidente pour l'homme et c'est elle, également, qui assure le lien entre les générations et la famille plus étendue ${ }^{36}$. Les hommes sont donc largement dépendants de leur conjointe pour satisfaire leurs besoins affectifs et maintenir leur santé psychologique et physique ${ }^{37}$. L'homme perd beaucoup plus lors de la séparation amoureuse, d'autant plus qu'il ne dispose pas d'un réseau social suffisamment solide et diversifié pour l'aider à se remettre sur pied.

\section{L'homosexualité}

Dans une société traditionnellement homophobe, la divulgation de l'homosexualité semble être un moment critique pour les jeunes hommes. Plusieurs études estiment que les jeunes hommes homosexuels sont jusqu'à 13 fois plus à risque de faire une tentative de suicide que les jeunes hommes hétérosexuels ${ }^{38}$. Comme le souligne Lisak $^{39}$, la masculinité se définit d'abord par ce qu'elle doit exclure, c'est-à-dire-la féminité. Ainsi, pour le jeune homosexuel et plus particulièrement le jeune homosexuel efféminé, la quête d'identité s'avère particulièrement difficile; le film Ma vie en rose 40 en est une belle illustration. D'ailleurs, le plus important facteur pour prédire l'état de santé mentale des jeunes homosexuels est l'acceptation de soi ${ }^{41}$. Ainsi, une orientation sexuelle hors norme constituerait en soi un facteur de risque $\mathrm{du}$ suicide, surtout pour les hommes puisque, dans notre société, elle entraîne un ostracisme qui affecte la qualité de vie des personnes qui en sont victimes.

\section{FACTEURS DE PROTECTION DU SUICIDE \\ CHEZ LES HOMMES}

\section{L'expression de la souffrance}

La souffrance fait partie de tous les scénarios suicidaires, qu'ils soient féminins ou masculins. Mais dans le cas des hommes, la souffrance est plus malaisément exprimée. En exigeant stoïcisme, indépendance et invulnérabilité, la socialisation masculine limite l'expression émotionnelle des hommes. Elle ne les autorise qu'à exprimer des émotions qui leur permettent de garder ou de reprendre le pouvoir et le contrôle sur les autres et sur eux-mêmes, par exemple: la colère et la jalousie. Si la souffrance des hommes ne peut se manifester par la dépression, le repli ou les pleurs, elle trouvera son exutoire dans l'agir, le travail compulsif, l'alcoolisme, la violence. D'ailleurs, le suicide ne serait-il pas une tentative ultime de reprise de contrôle sur la vie afin de mettre fin à une souffrance intolérable?

Puisque, trop souvent, les hommes expriment leur détresse par un agir impulsif et colérique, leur demande d'aide est mal interprétée par les proches et les intervenants, ce qui diminue considérablement l'efficacité des réseaux de soutien $^{42}$. En effet, une souffrance non dite ou mal exprimée est plus difficilement reconnue et, par conséquent, beaucoup plus difficile à soulager. Ainsi, les hommes obtiennent plus difficilement des services adaptés à leurs besoins parce que leurs signes de détresse sont moins bien décodés et reconnus.

\section{Les réseaux de soutien}

Lorsqu'on traverse une crise suicidaire, il est important d'avoir des amis ou de la famille qui puissent nous aider à surmonter nos difficultés. Or, les hommes disposent d'un réseau social moins riche que celui des femmes, en bonne partie à cause du meilleur soutien émotif de celles-ci ${ }^{43}$. L'Enquête sociale et de santé 1992-199344 nous apprend que les hommes sont plus nombreux que les femmes à avoir un faible niveau de soutien social, à n'avoir aucun confident ou personne pour leur témoigner de l'affection. Les hommes rapportent également faire moins souvent de rencontres sociales, avoir une plus faible satisfaction dans les rapports avec les amis et un nombre moins important de personnes disponibles en cas de besoin. Ces constats sont alarmants puisque le manque de soutien et une participation sociale réduite menacent le bien-être psychologique et physique et augmentent considérablement les probabilités de détresse psychologique, d'idéations suicidaires et de tentatives de suicide ${ }^{45}$.

La socialisation masculine serait en grande partie responsable de ce manque de soutien émotif dont souffrent les hommes. Les femmes sont socialisées dans des rôles expressifs et développent ainsi une compétence dans le domaine du soutien, alors que les hommes sont plutôt socialisés dans des rôles instrumentaux, ce qui ne leur permet pas de développer ce type de compétence ${ }^{46}$. D'ailleurs, dans leurs amitiés, les femmes mettent surtout l'accent sur la discussion et le partage d'émotions, alors que les hommes investissent davantage dans le partage d'activités ${ }^{47}$.

Les hommes ont également plus de difficulté que les femmes à demander de l'aide, notamment en raison de l'incompatibilité de ce comportement avec les attributs masculins de la compétence, $\mathrm{du}$ succès et de la réalisation de soi ${ }^{48}$. Ce n'est qu'après une longue période de dissimulation et de négation des symptômes, aussi sérieux soient-ils, que les hommes finiront par avouer l'inavouable: qu'ils ont un problème. Jusqu'à ce moment décisif où la demande d'aide pourra être envisagée, les mécanismes d'adaptation qu'ils utiliseront seront autant de modalités de fuite et de négation du problème, comme l'isolement, le repli sur soi, les pensées suicidaires, voire même le passage à l'acte ${ }^{49}$. 


\section{Les possibilités d'avenir}

À mesure que s'améliore le statut de la femme dans nos sociétés, les jeunes femmes peuvent envisager l'avenir avec plus d'optimisme, ce qui les protégerait contre le suicide ${ }^{50}$. Elles peuvent accéder à des postes intéressants qui leur étaient jadis interdits, elles fréquentent les universités en plus grande proportion que les hommes et un large éventail de possibilités s'ouvrent à elles. Par contre, la masculinité traverse actuellement une crise importante, dont les principales manifestations sont la perte de certains privilèges (dont celui d'unique pourvoyeur de la famille), les nouvelles attentes sociales (dont celle d'être très engagé dans le développement des enfants) et le traitement négatif des hommes dans les médias ${ }^{51}$. D'ailleurs, dans une étude réalisée par Germain Dulac $^{52}$, les hommes mentionnent leur désarroi à l'égard des images négatives de la masculinité véhiculées par la culture, particulièrement celles du mâle immoral et du parent toxique. Selon lui, la masculinité est actuellement constituée de comportements répréhensibles et indésirables. Les hommes provenant de divers milieux socio-économiques font état d'un malaise profond et ressentent une honte à être un homme. Cette crise identitaire les rend particulièrement vulnérables aux changements sociaux et personnels et probablement aussi au suicide.

\section{MULTIFACTORIALITÉ ET UNICITÉ DU SUICIDE}

Aucun des facteurs présentés ici n'agit de façon isolée. Au contraire, le suicide doit être considéré dans un cadre multidimensionnel où le risque suicidaire découle de l'interaction complexe de facteurs de risque, de facteurs de protection et d'éléments déclencheurs du comportement suicidaire. Linteraction des facteurs peut non seulement ouvrir la voie au comportement suicidaire, mais aussi le précipiter ${ }^{53}$.

Cependant, le suicide est unique. Unique dans le sens que pour chaque individu, pour chaque homme, les événements de vie se combinent de façon idiosyncrasique. Il n'y a pas deux trajectoires de vie identiques, ni deux scénarios suicidaires qui soient pareils. Ainsi pour chaque suicide, les facteurs de risque décrits plus haut se combinent d'une manière singulière pour pousser, chaque jour, trois hommes québécois à s'enlever la vie.

\section{QUE FAIRE?}

Dans la deuxième partie de cet article, nous avons constaté que la socialisation joue un rôle déterminant dans la trajectoire suicidaire des hommes. S'il en est ainsi, c'est d'abord à la socialisation que toute action préventive devrait s'adresser. Il faudrait alors s'attaquer tant aux rôles sexuels masculins (comportements, attitudes et conditionnements) et aux stéréotypes (idée que la plupart des gens se font du rôle masculin) qu'à l'idéal masculin (croyances de ce que devrait être l'homme idéal). Il s'agit là d'un mouvement de société qui nécessite une large mobilisation, voire une révolution.

Afin d'amorcer «tranquillement» cette révolution, la troisième partie de cet article se veut une invitation à réfléchir à nos pratiques et à nos attitudes face aux hommes en difficulté. Elle se base sur les résultats du projet de recherche que l'Action intersectorielle pour le développement et la recherche sur l'aide aux hommes (AIDRAH) a mené conjointement avec le chercheur Germain Dulac ${ }^{54}$.

\section{LE PARADOXE DE L'INTERVENTION}

Comme le rapporte Dulac, il existe un écart entre le modèle thérapeutique traditionnel et les compétences masculines. Ainsi, tel qu'indiqué au Tableau 3, beaucoup d'approches thérapeutiques exigent des clients certaines capacités qui entrent en contradiction avec les exigences de la masculinité.

Le monde thérapeutique demande aux hommes de renoncer à certains comportements et mécanismes d'adaptation, pour en adopter de nouveaux (par exemple, reconnaître l'échec au lieu du succès, utiliser la coopération au lieu de la compétition et la vulnérabilité au lieu du pouvoir). Ces contradictions font ressurgir les différences entre la socialisation masculine et les attentes de plusieurs thérapeutes et intervenants formés de manière traditionnelle. Dans un tel contexte, on peut mieux comprendre la réticence de certains hommes à demander de l'aide.

\section{LA POLITIQUE DE LA MAIN TENDUE}

Des intervenants œuvrant dans des organismes en prévention du suicide ont observé que ce sont souvent des proches (ami, conjointe, mère) de l'homme suicidaire qui appellent pour demander de l'aide. Après leur avoir donné de l'information, les intervenants demandent que l'homme suicidaire appelle lui-même. Or, près de la moitié des hommes n'appellent pas. Quelques intervenants ont choisi d'adopter une attitude plus proactive et, avec la permission des proches, ils contactent eux-mêmes le client suicidaire. Un tel appel est généralement très

Tableau 3

\section{Contradiction entre les exigences de la thérapie et de la masculinité55}

\section{Exigences de la thérapie}

- Dévoiler la vie privée

- Renoncer au contrôle

- Intimité non sexuelle

- Montrer ses faiblesses

- Faire l'expérience de la honte

- Être vulnérable

- Chercher de l'aide

- Exprimer les émotions

- Être introspectif

- S'attaquer aux conflits interpersonnels

- Confronter sa douleur, sa souffrance

- Reconnaître ses échecs

- Admettre son ignorance
Exigences de la masculinité

- Cacher la vie privée

- Maintenir le contrôle

- Sexualisation de l'intimité

- Montrer sa force

- Exprimer sa fierté

Être invincible

Être indépendant

Être stoïque

Agir, Faire

Éviter les conflits

Nier sa douleur, sa souffrance

Persister indéfiniment

Feindre l'omniscience 
bien reçu et il peut provoquer une excellente occasion de briser la barrière de la demande d'aide. La personne suicidaire ainsi rejointe est toujours libre de raccrocher, mais souvent, elle ne demande pas mieux que d'entrer en contact avec l'intervenant 56 .

Si cette pratique semble entrer en contradiction avec le principe de responsabilisation qui exige que ce soit le client lui-même qui fasse la démarche de demande d'aide, elle permet de contourner l'obstacle majeur de la réticence à rechercher de l'aide. Une fois le contact établi entre l'homme suicidaire et la l'aidant, il est toujours temps de le responsabiliser face à sa situation.

\section{RÉPONDRE PROMPTEMENT}

\section{A LA DEMANDE D'AIDE}

Les hommes qui ont consenti à faire les premières démarches pour obtenir de l'aide risquent de changer d'idée et de retourner à leur solitude, si leur demande n'est pas promptement accueillie. Cela pourra ensuite être très long avant que l'on puisse rétablir un lien de confiance avec eux. Par exemple, ils pourraient croire erronément qu'un rendez-vous reporté à plus tard confirme que leur situation n'est pas aussi grave qu'ils l'auraient cru. Les anciens mécanismes d'adaptation reprendront le dessus $^{57}$ et la personne aura encore plus de difficulté à se situer face à son problème.

Quand un homme en difficulté se décide à contacter quelqu'un pour demander de l'aide et qu'il compose enfin le numéro de téléphone, qu'il conserve peut-être depuis des mois, d'un organisme dont il a entendu parler... il ne doit surtout pas tomber sur un répondeur. Il est fort probable qu'il ne rappellera pas. Par contre, s'il trouve au bout $\mathrm{du}$ fil quelqu'un qui est attentif, le contact qui s'établira pourra être bénéfique: l'homme en a gros sur le coeur et depuis longtemps.

\section{DÉCODER LA DEMANDE D'AIDE}

$\mathrm{Au}$ Québec, depuis les années 1970, le taux de suicide des hommes a bondi de $78 \%$ et maintenant, $80 \%$ des suicides sont commis par des hommes. Comment expliquer l'ampleur de ce phénomène social? Notre analyse montre que la socialisation masculine joue un rôle déterminant. Elle facilite le développement de comportements et d'attitudes qui rendent les hommes plus vulnérables au suicide, tout en inhibant ceux qui pourraient les protéger. Force est de constater que depuis la fin des années 1960 , les rôles masculins ont été vigoureusement critiqués sans pour autant que les hommes se voient offrir de nouveaux modèles. En effet, ce sont encore les super héros masculins qui font entrer l'argent au box-office. Et ces super héros, adulés de tous, n'hésitent jamais à mettre leur vie en jeu pour sauver la patrie, la famille ou encore leur honneur.

Pourtant, les hommes qui s'enlèvent la vie, ce sont nos pères, nos frères, nos conjoints, nos collègues, nos amis, nos fils. Sans demander à la société québécoise de changer de façon drastique son regard et ses attentes face aux hommes, nous avons voulu ici amorcer une réflexion sur certains aspects de la condition masculine. La prévention du suicide chez les hommes passe par une reconnaissance sociale des effets pernicieux de leur socialisation et de l'extrême rigidité du rôle masculin. Nous devons, hommes et femmes, modifier nos façons de concevoir, de comprendre et d'accepter la souffrance au masculin. C'est sur cette base qu'une véritable mobilisation pourra s'organiser et mettre un frein à la progression alarmante du taux de suicide chez les hommes.

\section{Notes}

1 Danielle ST-LAURENT, "Analyse statistique du suicide chez les hommes adultes», dans Le dossier hommes et suicide de la Semaine provinciale de prévention du suicide, sous la direction de Lucie CHARBONNEAU, Montréal, Association québécoise de suicidologie, 1999, p. 5-7.

2 Ibid.

3 Sylvia S. CANETTO et Isaac SAKINOFSKY, «The Gender Paradox in Suicide», Suicide and Life-Threatening Behavior, vol. 28, no 1, 1998, p. 1-23.

4 Richard BOYER et al., L'épidémiologie des parasuicides et des idéations suicidaires, Montréal, Ministère de la santé et des services sociaux, 1992.

5 Marsha M. LINEHAN, "Suicide and Attempted Suicide: Study of Perceived Sex Differences», Perceptual and Motor Skills, vol. 37, 1973, p. 31-34.

6 Sylvia S. CANETTO, «Gender Roles, Suicide Attempts, and Substance Abuse», Journal of Psychology, vol. 125, 1991, p. 605-620; "Gender and Suicide in the Elderly», Suicide and Life-Threatening Behavior, vol. 22, 1992, p. 80-97.

7 Judith M. STILLION, «Ageism and Sexism in Suicide Attitudes», Death Studies, vol. 13, 1989, p. 247-261.

8 Brian L. MISHARA, "College Students Experiences with Suicide and Reactions to Suicidal Verbalizations», ibid.

9 Hedy WHITE et Judith M. STILLION, «Sex Differences in Attitudes Toward Suicide: Do Males Stigmatize Males?», Psychology of Women Quarterly, vol. 12, 1988, p. 357-372.

10 Alexander R. RICH et al., "Gender Differences in the Psychological Correlates of Suicidal Ideation Among Adoles- cents», Suicide and Life-Threatening Behavior, vol. 22, no 3, 1992, p. 364-373.

11 Robin J. LEWIS et George SHEPEARD, «Inferred Characteristics of Successful Suicide as Function of Gender and Context», Suicide and Life-Threatening Behavior, vol. 22, 1992, p. 187-198.

12 Sylvia S. CANETTO et Isaac SAKINOFSKY, «The Gender Paradox in Suicide», ibid.

13 D. A. COUNTS, «Fighting Back Is Not the Way: Suicide and the Women of Kaliai», American Ethologist, vol. 7, 1980 p. 332-351.

D. H. RUBINSTEIN, «Love and Suffering: Adolescent Socialization and Suicide in Micronesia», The Contemporary Pacific, vol. 7, no 1, 1995, p. 364 373.

14 Sylvia S. CANETTO et David LESTER, "Gender, Culture and Suicidal Behavior», Transcultural Psychiatry, vol. 35, no 2, 1998, p. 163-190.

15 Hillary M. LIPS, Sex and Gender: An Introduction, Mountain View, Mayfield Publishing Company, 1993.

16 David LESTER, Why People Kill Themselves: A 1990s Summary of Research Findings on Suicidal Behavior, Springfield, Charles C. Thomas, 1992.

Robert PLUTCHIK, «Suicide and Violence: The Two-Stage Model of Countervailing Forces», dans Suicide: Biopsychosocial Approaches, Alexander J. BOTSIS, Constantin R. SOLDATOS et Costas N. STEFANIS (dir. publ.), Amsterdam, Elsevier, 1997, p. 115-127.

17 David A. BRENT, «Personality Disorder, Personality Traits, Impulsive Violence, and Completed Suicide in Adolescents», Journal of American Academy of Child and Adolescent Psychiatry, vol. 33, no 8, 1994, p. $1080-1086$.

Jody KASHDEN et al., "Impulsivity in Suicidal and Nonsuicidal Adolescents», Journal of Abnormal Child Psychology, vol. 21, no 3, 1993, p. 339-353.

18 Matti VIRKKUNEN, «Personality Profiles and State Aggressiveness in Finnish Alcoholic, Violent Offenders, Fire Setters, and Healthy Volunteers», Archives of General Psychiatry, vol. 51, 1994 , p. $28-33$

19 E. COCCARO, "Serotonergic studies in patients with affective and personality disorders», Archives of General Psychiatry, vol. 46, 1989, p. 587-599.

20 Allan APTER, Robert PLUTCHIK et Herman M. VAN PRAAG, "Anxiety, Impulsivity and Depressed Mood in Relation to Suicidal and Violent Behavior», Acta Psychiatrica Scandinavica vol. 87, 1993, p. 1-5.

21 Sylvia S. CANETTO et Isaac SAKINOFSKY, «The Gender Paradox in Suicide», ibid.

22 Ibid

23 Sylvia S. CANETTO et David LESTER, «Gender, Culture and Suicidal Behavior», ibid.

24 Hillary M. LIPS, Sex and Gender: An Introduction, ibid.

25 Ibid. 


\section{AH! J'AI PAS PU MOURIR}

LE SUICIDE MANQUÉ*

De mourir j'ai pas d'chance. Ah! Que c'est donc choquant! Un jour, j'ai voulu m'pendre, Je vais vous dire comment : Je m'enfuis à la grange, Je voulus m'étrangler, La corde était trop tendre, Je n'ai pas pu mourir. Ah! J'ai pas pu mourir.

De là, je pars de suite, M'enfuis chez l'pharmacien Chercher du poison vive Et puis, je m'en reviens, Je prends toute la dose, Décidé d'en finir.

C'était de l'eau de rose, Je n'ai pas pu mourir. Ah! j'ai pas pu mourir.

Pour y mieux réussir, Je prends mon pistolet; De moi vous allez rire Comme d'un vrai beignet. J'le bourre à ma manière C'est pour y moins souffrir, J'mets la balle la première Je n'ai pas pu mourir. Ah! j'ai pas pu mourir.

Enfin, pour une dernière Mille, nom d'un tonneau! J'm'en vas à la rivière Pour me jeter à l'eau, Sur le pont de Milèche, Là je pouvais choisir. J'me jette où c'était sèche, Je n'ai pas pu mourir. Ah! J'ai pas pu mourir.

Enfin, sur cette terre Il faut donc y rester; Pour vivre à la galère, Y vivre sans gaieté. Si la mort vient me prendre, Lui dirai sans mentir: «Il n'est plus temps d'me prendre, Je ne veux plus mourir. Ah! Ne veux plus mourir»

* Collection Adélard Lambert, ms no 12, s.d., chanté par Adélard Lambert, St-Cuthbert, Berthier, Québec.

Fonds du CELAT, Université Laval. Fonds Luce Des Aulniers.
26 Sylvia S. CANETTO, «Gender Roles, Suicide Attempts, and Substance Abuse», ibid.

27 Louise GUYON et al., "Grande consommation d'alcool et problèmes connexes», dans Aspects sociaux reliés à la santé: Rapport de l'Enquête sociale et de santé 1992-1993, vol. 2, sous la direction de C. LAVALLÉE et al., Montréal, Ministère de la santé et des services sociaux, 1995, p. 81-112.

28 Michel TOUSIGNANT et Tyna PAYETTE, Suicide et toxicomanie: deux phénomènes interreliés, Montréal, Conseil permanent de lutte à la toxicomanie, 1997.

29 Stephen PLATT, «Unemployment and Suicidal Behavior: A Review of the Literature», Social Science and Medicine, vol. 19, 1984, p. 93-115.

Colin PRITCHARD, "Is There a Link Between Suicide in Young Men and Unemployment Among Men in Edinburg», British Journal of Psychiatry, vol. 160, 1992, p. $750-756$.

30 D. J. LEVINSON, "A Conception of Adult Development», American Psychologist, vol. 41, 1986, p. 3-13.

31 G. H. EDWARDS, "The Structure and Content of the Male Gender Role Stereotype: An Exploration of Subtypes», Sex Roles, vol. 27, 1992, p. 533-551. E. M. ENGLAND, "College Student Gender Stereotypes: Expectations about the Behavior of Male Subcategory Members», Sex Roles, vol. 27, 1992, p. 699-716.

D. R. MCCREARY et S. W. SAVADA, «Mediating the Relationship Between Masculine Gender Role Stress and Work Satisfaction: The Influence of Coping Strategies», The Journal of Men's Studies, vol. 4, no 2, 1995, p. 141-152.

32 Germain DULAC, Les demandes d'aide des hommes, Montréal, Centre d'études appliquées sur la famille, 1997.

Keith HAWTON, "Why Has Suicide Increased in Young Males?», Crisis, vol. 19, no 3, 1998, p. 119-124.

33 Ibid.

34 Jean-François SAUCIER, «Pourquoi les hommes se suicident-ils plus que les femmes?», dans Le dossier hommes et suicide de la Semaine provinciale de prévention $d u$ suicide, sous la direction de Lucie CHARBONNEAU, Montréal, Association québécoise de suicidologie, 1999, p. 19-22.

35 Nancy CHODOROW, The Reproduction of Mothering, Berkeley, University of California Press, 1980.

36 Germain DULAC, Les demandes d'aide des hommes, ibid.

37 Ibid.

38 Pierre TREMBLAY et Richard RAMSAY, «Gay and Bisexual Male Youth: Overrepresented in Suicide Problems and Associated Risk Factors» Présentation à la Troisième conférence bi-régionale sur la prévention du suicide chez les adolescents, Colorado, 1997, p. 21-23. Cette présentation est disponible sur le site: http://www.virtualcity.com/youthsuicide/
39 David LISAK, «Integrating a Critique of Gender in the Treatment of male survivors of Chilhood Abuse», Psychotherapy, vol. 32, no 2, 1995, p. 258-269.

$40 \mathrm{Ma}$ vie en rose, film réalisé par Alain Berliner, Belgique-France, 1997.

41 Pierre TREMBLAY et Richard RAMSAY, «Gay and Bisexual Male Youth: Overrepresented in Suicide Problems and Associated Risk Factors», ibid.

42 David LISAK, "Integrating a critique of Gender in the Treatment of male survivors of Chilhood Abuse», ibid.

Germain DULAC, Intervenir auprès des clientèles masculines: théories et pratiques québécoises, ibid.

43 Jocelyne CAMIRAND, R. MASSÉ et Michel TOUSIGNANT, «Milieu social», dans Aspects sociaux reliés à la santé: Rapport de l'Enquête sociale et de santé 1992-1993, vol. 2, Montréal, Ministère de la santé et des services sociaux, 1995, p. 125-141.

44 Ibid.

45 R. MASSÉ et C. POULIN, «Mental Health of Community Residents in the Metropolitan Area: Some Results of the "Santé Québec Survey»», Canadian Journal of Public Health, vol. 82, no 5, 1991, p. 320-324.

46 Germain DULAC, Les demandes d'aide des hommes, ibid.

47 M. A. CALDWELL et L. A. PEPLAU, "Sex Differences in Same-Sex Friendships», Sex Roles, vol. 8, 1982, p. 721-732.

48 Germain DULAC, Les demandes d'aide des hommes, ibid.

49 Ibid.

50 Francine GRATTON, "Le climat social du Québec, propice à des suicides d'“être" chez les jeunes?», P.R.I.S.M.E., vol. 5, no 4, 1995, p. 510-523.

51 R. F. LEVANT, «The Masculinity Crisis», The Journal of Men's Studies, vol. 5, no 3, 1997, p. 221-231.

52 Germain DULAC, Les demandes d'aide des hommes, ibid.

53 Ronald DYCK, Brian MISHARA et Jennifer WHITE, «Le suicide chez les enfants, les adolescents et les personnes âgées: constatations clés et mesures préconisées» dans Forum National sur la Santé: Les Déterminants de la Santé vol. 3: Le Cadre et les Enjeux, 1998, p. 323-390.

54 Germain DULAC, Intervenir auprès des clientèles masculines: théories et pratiques québécoises, ibid.

55 SHAY, 1996, cité dans DULAC, 1999, p. 13.

56 Germain, DULAC et Lucie CHARBONNEAU, "Intervenir auprès des hommes en difficulté», dans Le dossier hommes et suicide de la Semaine provinciale de prévention du suicide, sous la direction de Lucie CHARBONNEAU, Montréal, Association québécoise de suicidologie, 1999, p. 35-42

57 Ibid. 